\title{
EDITORIAL
}

\section{Immunity check should be performed for all patients with septic shock? Yes}

\author{
Julien Textoris ${ }^{*}$ (])
}

(c) 2020 Springer-Verlag GmbH Germany, part of Springer Nature

Septic shock is the most severe form of sepsis, i.e., an infection associated with organ failures, due to a dysregulated host response [1]. Among the body systems that respond in a dysregulated way, the immune system plays a major role in the pathophysiology of sepsis. Indeed, although a pathogen is responsible for the infection, and will trigger the host immune response, the organ failures are the consequence of this inappropriate immune response. This has been demonstrated nicely, amongst other, by Poltorak et al. [2]. Therefore, as we use diagnostics to manage infection (ID/AST ${ }^{1}$ ), we should monitor the immune response if we wish to act on it.

Our current understanding of the host immune response and the pathophysiology of sepsis is described in Fig. $1[3,4]$. This complex interplay between the various components of the immune response is highly dynamic, underlying the need for monitoring to understand where a given patient stands on such altered response. Our understanding that such response is altered comes mainly from observations that in septic patients, the level of plasma cytokines (such as IL-6 or IL-10) or other phenotypic markers (e.g., altered expression of HLA-DR or lymphopenia) is 100 - to 1000 -folds what is seen in a physiological response. Moreover, many of these immune-related biomarkers have been associated with relevant clinical endpoints such as mortality or the occurrence of secondary infections [5-10]. Multiple experimental and animal studies also provided

\footnotetext{
${ }^{1}$ IDentification of the pathogen responsible for the infection, and Antibiotic Susceptibility Testing (AST).
}

*Correspondence: julien.textoris@gmail.com EA7426 "Pathophysiology of Injury-Induced Immunosuppression", PI3, Université Claude Bernard Lyon-1, Hospices Civils de Lyon, bioMérieux, Lyon, France supporting evidences that manipulating the host immune response could allow (1) to avoid or treat organ failures (mostly blocking the pro-inflammatory side of the response), and (2) avoid secondary infections and promote healing (nicely reviewed in [3, 4]). Most attempts to translate this into clinical practice have failed, mainly because (1) we did not take into account the dynamics of the response (as mentioned above), thus administering a drug at the wrong time (e.g., TNF inhibitor once the patient is already exhibiting immune-paralysis), and (2) we ignored which threshold should triggered the administration of an immune-modulatory drug. Measuring some immune parameters and defining such threshold should, therefore, help us to select among all the potential immune-modulatory alternatives (such as GM-CSF, IFNG-g, checkpoint inhibitors, or IL-7), and promote the most promising ones.

The third reason to support immune monitoring is to acknowledge the heterogeneity of the septic population [11]. Indeed, we have now several studies underlying that sepsis encompasses multiple phenotypes or endotypes. The fulminant hyperinflammatory response of a young children to meningococcemia is far different from a ventilator-associated pneumonia due to pseudomonas in a highly comorbid patient. A recent paper from Seymour et al. recently identified four clinical endotypes, and nicely shown that varying the proportion of these endotypes within a given trial population might change completely the result of such trial [12]. Similarly, Wong et al. and Antcliffe et al. have also shown that identifying subgroup of patients by transcriptional signatures was associated with different outcomes upon hydrocortisone treatment [13-15]. The ability to stratify septic patients (or more broadly, critically ill patients) into more homogeneous subgroups will be key to demonstrate the efficacy of immune manipulation strategies [16]. This has been suggested clinically by Meisel et al.with their

\section{6 Springer}




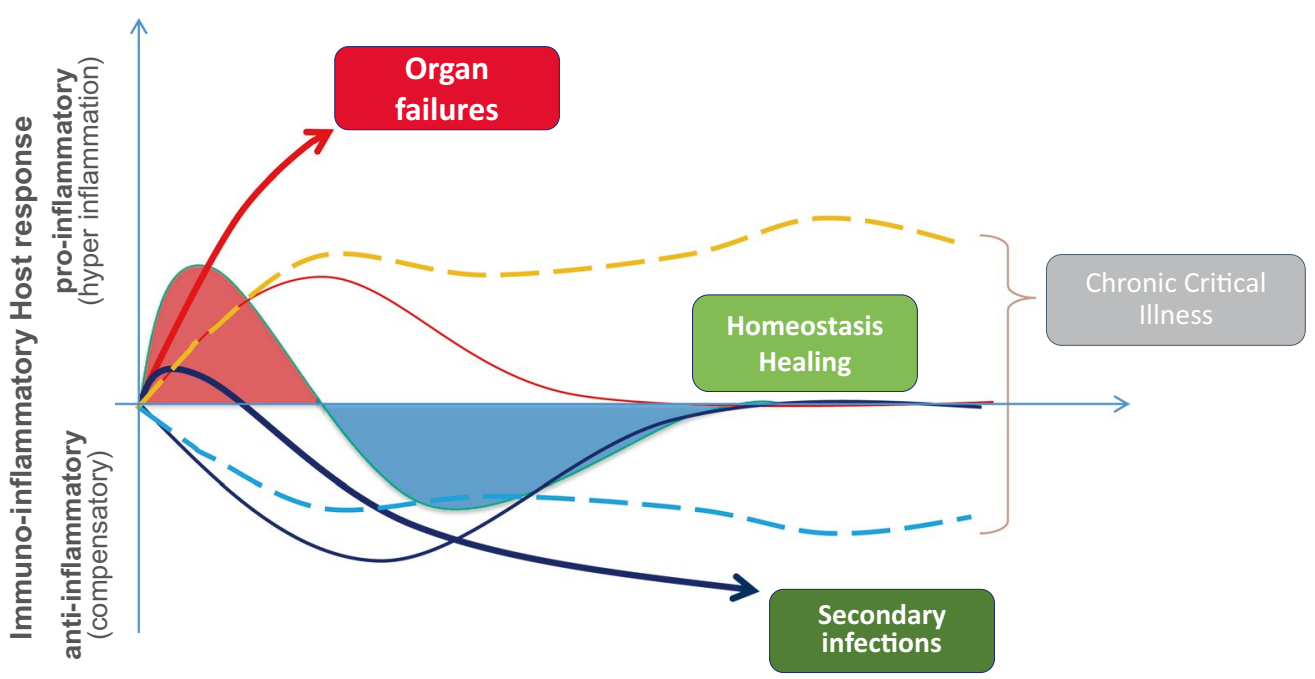

Fig. 1 Schematic representation of the host immune response in sepsis. Any time the body is injured, a host immune response is observed (which can be triggered by an infectious insult, or a sterile one such as trauma, burn, and surgery). When the invasive pathogen triggers the immune response, innate immune system will quickly react to control the proliferation and spread of the pathogen, and kill it. Both a pro-inflammatory and an anti-inflammatory response will start (thin red and blue lines). The net balance is towards pro-inflammation initially and then shift towards anti-inflammation (red and blue areas). These powerful mechanisms are also deadly for our own cells, and when exacerbated, will lead to organ failures, in particular, a severe cardio-vascular dysfunction (thick red line). To avoid us from dying any time we got infected, our immune system has elaborated compensatory mechanisms, that will help our immune system to quickly return to homeostasis and allow healing. However, when this compensatory response is overstated and prolonged (thick blue line), this translates into immune paralysis, which favors secondary infections, and impairs the return to homeostasis and healing processes. Over time, when no resolution of inflammation occurs, patients will evolve towards a chronic inflammatory state (with both features of the pro-inflammatory and anti-inflammatory response, dotted light blue, and orange lines) that is a key component of the chronic critical illness pathophysiology

pilot study on the effect of boosting the immune system by GM-CSF (the patients were stratified on the low expression of monocytic HLA-DR), or more recently by Shakoory et al. who demonstrated that macrophage activation syndrome could identify those that may benefit from the administration of recombinant IL-1b antagonism $[17,18]$. While, up to now, prognostic enrichment has been favored in septic shock, predictive enrichment may indeed be an interesting alternative [19].

To do so, unresolved issues are to determine which immune parameters are relevant, and which threshold would provide the best stratification performances. However, discriminating the normal immune response (which is physiologically different from homeostasis) from an immune dysfunction is difficult. Interestingly, Timmermans et al. used severe trauma patients to describe the initial host immune response to injury (because in trauma, the onset time is known precisely, and we know that the host immune response to injury is similar to what is seen in sepsis). They showed that as soon as $\mathbf{3 0}$ min after trauma, many immune-related biomarkers were already altered, such as the inability to produce TNF after ex-vivo stimulation by LPS [20]. Such very early response most probably reflects the physiological compensatory response. However, as patients exhibiting a decrease in HLA-DRA expression between admission and day 3 (ratio $<1$ ) were more likely to develop secondary infections, it is more the depth and duration of these alterations that we should call immune suppression. Our ability to understand when the host response shifts from a physiological response to a pathological one will be key. Moreover, given the complexity of the immune system, we need to understand if alterations on the innate and adaptive side of the immune response are sequential or concomitant, and if they both contribute, additively or synergistically to the risk of secondary infections and mortality. A recent study by Conway-Morris et al. demonstrated that a combination of flow cytometry-based biomarkers could help to assess for adverse outcomes [10]. However, flow cytometry has some limitations (availability, standardization, etc), and assessing the transcriptional immune response (e.g., with a multiplex PCR tool) is probably an interesting alternative to explore the immune response. The REALISM study should provide some answers to these points soon, as it included several hundred ICU patients, and monitored numerous biomarkers covering all facets of the immune system (phenotypic, soluble markers, transcriptomic markers, and functional assays) over 2 months after ICU admission [21]. This should help us 
to better capture the aforementioned heterogeneity, and identify which biomarker combination will allow a better patient stratification.

In summary, we do need an immunoscope, i.e., a way to monitor quantitatively all key aspects of the immune response overtime, in ICU, because the host immune response (1) is clearly responsible for the pathophysiology of sepsis and septic shock, (2) is highly dynamic, (3) but also heterogeneous among patients, and (4) its complexity (number of players, and complex interplay of immune functions) prevent the use of a single biomarker. The ability to assess the immune status of critically ill patients will facilitate the adoption of adaptive clinical trials, allowing enrichment and stratification of patients who will be helped most by specific management approaches and treatments [22]. It is quite disappointing to have reached such detailed knowledge about the role of the immune system in critical illness, without having translated that into clinical practice. Immune failure should be part of the SOFA score, besides other key organ failures. Checking the immune status of septic shock patients will be the only way to demonstrate efficacy of immune manipulation strategies, and decrease mortality of such syndrome, as well as fighting against antimicrobial resistance.

\section{Compliance with ethical standards}

\section{Conflicts of interest}

Julien Textoris is a part-time employee of an in-vitro diagnostic company, bioMérieux, and part-time employee of Hospices Civils de Lyon. Both institutions hold patents related to sepsis biomarkers and JT is inventor on some of them. The views presented in this editorial are JT's personal opinion and do not necessarily represent the viewpoint, strategy, or opinions of bioMérieux.

\section{Publisher's Note}

Springer Nature remains neutral with regard to jurisdictional claims in published maps and institutional affiliations.

Received: 16 October 2019 Accepted: 19 December 2019 Published online: 21 January 2020

\section{References}

1. Singer M, Deutschman CS, Seymour C et al (2016) The third international consensus definitions for sepsis and septic shock (sepsis-3). JAMA 315:801-810. https://doi.org/10.1001/jama.2016.0287

2. Poltorak A, He X, Smirnova l et al (1998) Defective LPS signaling in C3H/ $\mathrm{HeJ}$ and C57BL/10ScCr mice: mutations in T/r4 gene. Science 282:20852088. https://doi.org/10.1126/science.282.5396.2085

3. Hotchkiss RS, Monneret G, Payen D (2013) Sepsis-induced immunosuppression: from cellular dysfunctions to immunotherapy. Nat Rev Immunol 13:862-874. https://doi.org/10.1038/nri3552

4. van der Poll T, van de Veerdonk FL, Scicluna BP, Netea MG (2017) The immunopathology of sepsis and potential therapeutic targets. Nat Rev Immunol advance online publication. https://doi.org/10.1038/nri.2017.36
5. Calandra T, Gerain J, Heumann D et al (1991) High circulating levels of interleukin-6 in patients with septic shock: evolution during sepsis, prognostic value, and interplay with other cytokines. The Swiss-Dutch J5 Immunoglobulin Study Group. Am J Med 91:23-29. https://doi. org/10.1016/0002-9343(91)90069-a

6. Peronnet E, Venet F, Maucort-Boulch D et al (2017) Association between mRNA expression of CD74 and IL 10 and risk of ICU-acquired infections: a multicenter cohort study. Intensive Care Med 43:1013-1020. https://doi. org/10.1007/s00134-017-4805-1

7. Monneret G, Lepape A, Voirin N et al (2006) Persisting low monocyte human leukocyte antigen-DR expression predicts mortality in septic shock. Intensive Care Med 32:1175-1183. https://doi.org/10.1007/s0013 4-006-0204-8

8. Landelle C, Lepape A, Voirin N et al (2010) Low monocyte human leukocyte antigen-DR is independently associated with nosocomial infections after septic shock. Intensive Care Med 36:1859-1866. https:// doi.org/10.1007/s00134-010-1962-x

9. Drewry AM, Samra N, Skrupky LP et al (2014) Persistent lymphopenia after diagnosis of sepsis predicts mortality. Shock 42:383-391. https://doi. org/10.1097/SHK.0000000000000234

10. Conway Morris A, Datta D, Shankar-Hari M et al (2018) Cell-surface signatures of immune dysfunction risk-stratify critically ill patients: INFECT study. Intensive Care Med 44:627-635. https://doi.org/10.1007/s0013 4-018-5247-0

11. Marshall JC (2014) Why have clinical trials in sepsis failed? Trends Mol Med 20:195-203. https://doi.org/10.1016/j.molmed.2014.01.007

12. Seymour CW, Kennedy JN, Wang S et al (2019) Derivation, validation, and potential treatment implications of novel clinical phenotypes for sepsis. JAMA 321:2003-2017. https://doi.org/10.1001/jama.2019.5791

13. Wong HR, Atkinson SJ, Cvijanovich NZ et al (2016) Combining prognostic and predictive enrichment strategies to identify children with septic shock responsive to corticosteroids. Crit Care Med 44:e1000-1003. https ://doi.org/10.1097/CCM.0000000000001833

14. Wong HR, Cvijanovich NZ, Anas N et al (2018) Endotype transitions during the acute phase of pediatric septic shock reflect changing risk and treatment response. Crit Care Med 46:e242-e249. https://doi. org/10.1097/CCM.0000000000002932

15. Antcliffe DB, Burnham KL, Al-Beidh F et al (2019) transcriptomic signatures in sepsis and a differential response to steroids. From the VANISH randomized trial. Am J Respir Crit Care Med 199:980-986. https://doi. org/10.1164/rccm.201807-14190C

16. Shankar-Hari M, Rubenfeld GD (2019) Population enrichment for critical care trials: phenotypes and differential outcomes. Curr Opin Crit Care 25:489-497. https://doi.org/10.1097/MCC.0000000000000641

17. Meisel C, Schefold JC, Pschowski R et al (2009) Granulocyte-macrophage colony-stimulating factor to reverse sepsis-associated immunosuppression. Am J Respir Crit Care Med 180:640-648. https://doi.org/10.1164/ rccm.200903-03630C

18. Shakoory B, Carcillo JA, Chatham WW et al (2016) Interleukin-1 receptor blockade is associated with reduced mortality in sepsis patients with features of macrophage activation syndrome: reanalysis of a prior phase III trial. Crit Care Med 44:275-281. https://doi.org/10.1097/CCM.00000 00000001402

19. Pickkers P, van der Poll T (2018) "What's new?"Immunostimulating strategies in the ICU. Intensive Care Med. https://doi.org/10.1007/s0013 4-018-5462-8

20. Timmermans K, Kox M, Vaneker M et al (2016) Plasma levels of dangerassociated molecular patterns are associated with immune suppression in trauma patients. Intensive Care Med 42:551-561. https://doi. org/10.1007/s00134-015-4205-3

21. Rol M-L, Venet F, Rimmele T et al (2017) The REAnimation Low Immune Status Markers (REALISM) project: a protocol for broad characterisation and follow-up of injury-induced immunosuppression in intensive care unit (ICU) critically ill patients. BMJ Open 7:e015734. https://doi. org/10.1136/bmjopen-2016-015734

22. Textoris J, Gordon AC (2018) Sepsis: who will shoot first? Pharma or diagnostics? Intensive Care Med 44:1331-1333. https://doi.org/10.1007/s0013 4-018-5234-5 